\title{
A Comparison of Linear and Daily Undulating Periodized Strength Training Programes for Quadriceps Strength in Normal Young Male Population
}

\author{
Kulbir Singh Rana and Anuradha Lehri
}

\begin{abstract}
Aim: To determine more efficient method of periodized strength training program, and to determine if either method of periodization elicits superior gains in 1RM and $10 \mathrm{RM}$ for quadriceps muscle in males. Material and Method: A sample of thirty normal males aged between 18-30 years was taken for the study by convenient (purposive) sampling and were randomly divided into two groups consisting of thirty each i.e. Group-A (Trained with Linear periodization (LP) and Group-B (Trained with Daily undulating periodization (DUP).An experimental design involving the comparative analysis with pre and post-test scores of the two groups, namely Group-A \& B, was used in this study. The total duration of the study was 6 Weeks. Results: Comparison of means of pre-test score of $1 \mathrm{RM}$ shows that there is no significant difference between two groups i.e. same at baseline. Comparison of means of $1 \mathrm{RM}$ at 3 weeks and at 6 weeks shows no significant differences. Hence it showed that there are no significant improvements between groups analysis of $1 \mathrm{RM}$. Within group analysis with $1 \mathrm{RM}$ scores shows the significant change in strength when comparison of pre-test to 3 weeks and pre-test to 6 weeks was done. It indicates that the linear periodisation and daily undulating periodisation is effective in improving the strength of quadricep muscle. Comparison of means of pre-test score of $10 \mathrm{RM}$ shows that there is no significant difference between two groups i.e. same at baseline. Comparison of means of $1 \mathrm{RM}$ at 3 weeks and at 6 weeks shows no significant differences. Hence it showed that there is no significant improvement between groups analysis of $10 \mathrm{RM}$. Within group analysis with $10 \mathrm{RM}$ scores shows the significant change in strength when comparison of pre-test to 3 weeks and pre-test to 6 weeks was done. It indicates that the linear periodisation and daily undulating periodisation is effective in improving the strength of quadriceps muscle. Conclusion: The study concludes that both linear periodization and Daily undulating periodization are equally effective means of improving the strength and endurance of Quadriceps in males of age group 18$30 \mathrm{yrs}$.
\end{abstract}

Kulbir Singh Rana

Ph.D. Student

Department of Sports Science

Punjabi University, Patiala(Punjab) India

E mail: kbsrana@gmail.com

Anuradha Lehri

Assistant Professor

Department of Sports Science

Punjabi University, Patiala(Punjab) India

E-mail: anu_lehri@yahoo.com
Key Words: Periodisation, Quadriceps, Strength, Training, 1RM

DOI: $10.18376 / j e s p / 2019 / v 15 / i 1 / 111318$

\section{Introduction}

The popularity of resistance training has grown immensely over the past 25 years, with extensive research demonstrating that not only is resistance training an effective method to improve neuromuscular function, it can also be equally effective in maintaining or improving individual 


\section{Journal of Exercise Science \& Physiotherapy Vol. 15 No. 1 (January to June) 2019 \\ ISSN: 0973-2020 (Print) I I OR Impact Factor $=6.502 \quad$ ISSN: 2454-6089 (Online)}

health status. However, designing a resistance-training program is a complex process that incorporates several acute program variables and key training principles (Baechle, T. B., \& Earle, R. E. (2000).With the advent of modern technology,materials and knowledge, the methods that can be used for strength training have multiplied significantly. The resistance in these exercises can be applied in form of manual or mechanical resistance. Resistance training exercises can be performed isometrically, isotonically, and isokinetically to increase strength and endurance, other forms include plyometric training and circuit training for gains in muscular strength and endurance (Kraemer, W. J. (1997). Training variation requires that alterations in one or more program variables be made over time to allow for the training stimulus to remain optimal. It has been shown that systematically varying volume and intensity is most effective for long-term progression compared with programs that did not vary any acute program variable. The concept of variation has been part of program design for many years. The importance of training variation, or periodization, became apparent for resistance training as a result of the work of Canadian scientist, Dr. Hans Selye (Pedemonte, J. (1986). Strength training and conditioning plays a major role in normal subjects. Professionals are concerned with determining the most effective means for developing muscular strength. Variation or periodization of training is an important concept in designing weight-training programs. There are various types of the periodized programs the numerous configurations of the program variables and the effects of various periodization protocol often leads to a difficulty in decision making that what type of periodized program is the most effective.

Linear periodization (LP) is the traditional periodization model because of the gradual increases in training intensity and decreases in training volume over time. These changes are typically made approximately every four weeks (Graham, J. (2002); Brown, A. L. (2001).LP structures different training cycles into specific time periods based on a specific goal. Usually the main goals are hypertrophy, strength, and power (Baker, D., Wilson, G., \& Carlyon, R. (1994).

Non-linear periodization also referred to as undulating periodization, differs from linear periodization by making changes in intensity and volume on a more frequent basis, typically weekly or bi-weekly (Pedemonte, J. (1986); Brown, A. L. (2001).Daily undulating periodization (DUP) is also a non-linear system with alterations in training volume and intensity made on a daily basis (Brown, A. L. (2001); Baker et al (1994).

Daily Undulating Periodization

Linear Periodization
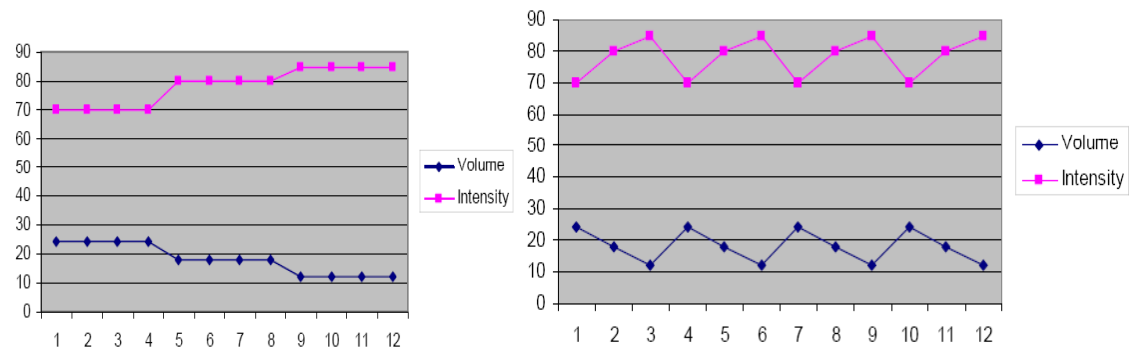

The purpose of study is to find more efficient method of periodized strength training program, and to determine if either method of periodization elicits superior gains in 1RM and 10 RM for quadriceps muscle in males.

\section{Material and Methods}

A sample of thirty normal males aged between 18-30 years was taken for the study by convenient (purposive) sampling and were randomly divided into two groups consisting of thirty each: 


\section{Journal of Exercise Science \& Physiotherapy Vol. 15 No. 1 (January to June) 2019 \\ ISSN: 0973-2020 (Print) I I OR Impact Factor $=6.502 \quad$ ISSN: 2454-6089 (Online)}

Group-A: Trained with Linear periodization (LP); Group-B: Trained with Daily undulating periodization (DUP).

An experimental design involving the comparative analysis with pre and post-test scores of the two groups, namely Group-A \& B, was used in this study. The total duration of the study was 6 Weeks. The subjects who met the inclusion and exclusion criteria were asked to complete a preparticipation health screen (PARQ). The potential subjects were then explained about the purpose of the study and written consent was taken seeking their participation and cooperation. A normal assessment was also taken for all the subjects including 1RM and $10 \mathrm{RM}$ to determine the strength and endurance for quadriceps. Then the subjects were randomly assigned to one of the two groups: Group-A (LP) or Group-B (DUP and were asked to attend an instructional training session to ensure that the proper technique is used throughout the study. Following pre-training testing and randomization, subjects underwent a 6-week resistance-training program; 3 days per week. Results of the 1RM and 10RM tests were used to develop a safe and effective resistance training prescription for each of the study participant. The subjects were prohibited from performing other strength-developing exercises during the span of the 6-week study.All strength training and testing sessions were supervised. Each session began and ended with the appropriate warm-up and cooldown. Subject did warm up by completing a number of sub maximal repetitions. 1 RM was determined within four trials with rest periods of 3-5 min between trials. The initial weight selected was within the subject's perceived capacity (50-70\% of capacity). Resistance was then progressively increased until the subject could not complete the selected repetitions. All repetitions were performed at the same speed of movement and range of motion to instill consistency between trials. The final weight successfully lifted was recorded as Absolute RM. After calculation of $1 \mathrm{RM}$ for strength, $10 \mathrm{RM}$ for endurance was determined (Stone et al (1981). All the subjects then performed following exercises.

Knee extension exercises/Seated knee extension: participant sat on the Quadriceps table, with the exercising weight. Placed front of ankle of reference leg under pad and slowly straighten knees, holding it, contracting the thigh muscles as tight as possible and slowly lowered and return to start position. Knee extension exercises in sitting position were also given to the non-reference extremity. Progression was done as per the group protocol.

Followed the LP protocol in which the subjects carried out 3 sets of prescribed exercise every session. For each session in $1^{\text {st }}$ and $2^{\text {nd }}$ week, 8 repetitions with $70 \%$ of 1 RM were performed, followed by 6 repetitions for the $3^{\text {rd }}$ and $4^{\text {th }}$ weeks; with $80 \%$ of $1 \mathrm{RM}$ and lastly 4 repetitions for the $5^{\text {th }}$ and $6^{\text {th }}$ weeks; with $85 \%$ of 1 RM.Followed the DUP protocol in which the subjects carried out 3 sets of prescribed exercise every session. For the entire 6 weeks, the subjects performed 8 repetitions for 1 st session of every week; with $70 \% \mathrm{RM}$ of $1 \mathrm{RM}, 6$ repetitions for $2^{\text {nd }}$ session of every week; with $80 \%$ of $1 \mathrm{RM}$ and 4 repetitions for $3^{\text {rd }}$ session for every week with $85 \%$ of $1 \mathrm{RM}$. Reassessments were taken mid-training (after 3 weeks) and post-training (at the end of 6 weeks) to determine the improvement in the parameters of strength and endurance of quadriceps muscle using the $1 \mathrm{RM}$ and $10 \mathrm{RM}$ values respectively.

Manual entry of the data was done on a pre-planned format. Individual record of each subject was noted in his own form. Data was statistically analysed by using software SPSS 17 (USA, Illinois) .Between the group analysis was done using unpaired t- test. For Within the group analysis Repeated measure ANOVA was used for analysis within the groups. Significance level of $p<0.05$ was fixed.

\section{Results and Discussion}

Thirty normal male subjects with a mean age of 22.43 years were selected for the study. The mean, variance and standard deviation values of the age in the study groups are shown in table1. The 


\section{Journal of Exercise Science \& Physiotherapy Vol. 15 No. 1 (January to June) 2019 \\ ISSN: 0973-2020 (Print) $\quad I_{2}$ OR Impact Factor $=6.502 \quad$ ISSN: 2454-6089 (Online)}

comparison of these variables showed that there is no significant difference between groups hence the demographic data was equal at baseline.

Table 1. Demographic data

\begin{tabular}{|c|c|c|}
\hline Study groups & $\mathbf{N}$ & Mean \pm sd \\
\hline Group-a & 15 & $22.53 \pm 2.95$ \\
\hline Group-b & 15 & $22.33 \pm 3.35$ \\
\hline Total & 30 & $22.43 \pm 3.15$ \\
\hline
\end{tabular}

Comparison of means of pre-test score of $1 \mathrm{RM}$ shows that there is no significant difference between two groups i.e. same at baseline. Comparison of means of $1 \mathrm{RM}$ at 3 weeks and at 6 weeks shows no significant differences. Hence it showed that there are no significant improvements between groups analysis of $1 \mathrm{RM}$. Within group analysis with $1 \mathrm{RM}$ scores shows the significant change in strength when comparison of pre-test to 3 weeks and pre-test to 6 weeks was done. It indicates that the linear periodisation and daily undulating periodisation is effective in improving the strength of quadricep muscle.

Table 2. Within and between group comparison for strength 1 RM

\begin{tabular}{|c|c|c|c|c|c|c|c|c|}
\hline & \multirow{2}{*}{$\begin{array}{l}\text { Pre Test } \\
\text { PT } 0\end{array}$} & \multirow{2}{*}{$\begin{array}{l}\text { Post test } 1 \\
\text { PT } 1\end{array}$} & \multirow{2}{*}{$\begin{array}{l}\text { Post test } 2 \\
\text { PT } 2\end{array}$} & \multicolumn{2}{|c|}{ ANOVA } & \multirow{2}{*}{$\begin{array}{l}\text { PT0 } \\
\text { VS } \\
\text { PT1 }\end{array}$} & \multirow{2}{*}{$\begin{array}{l}\text { PT0 } \\
\text { VS } \\
\text { PT2 }\end{array}$} & \multirow{2}{*}{$\begin{array}{l}\text { PT1 } \\
\text { VS } \\
\text { PT2 }\end{array}$} \\
\hline & & & & $\mathrm{F}$ & $\mathrm{P}$ & & & \\
\hline Group A & $10.80 \pm 2.244$ & $13.00 \pm 2.53$ & $15.33 \pm 2.76$ & 438.303 & 0.000 & .000 & .000 & .000 \\
\hline Group B & $11.53 \pm 1.92$ & $13.466 \pm 2.29$ & $15.00 \pm 2.50$ & 550.820 & 0.000 & .000 & .000 & .000 \\
\hline 5 & 0.740 & 0.740 & 0.932 & & & & & \\
\hline 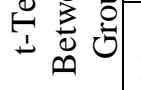 & 0.344 & 0.601 & 0.732 & & & & & \\
\hline
\end{tabular}

Keys: PT 0: Mean \pm SD of Pre-test scores of $1 \mathrm{RM}$; PT 1: Mean \pm SD of scores after three weeks for 1 RM; PT 2: Mean \pm SD of scores after six weeks for 1RM

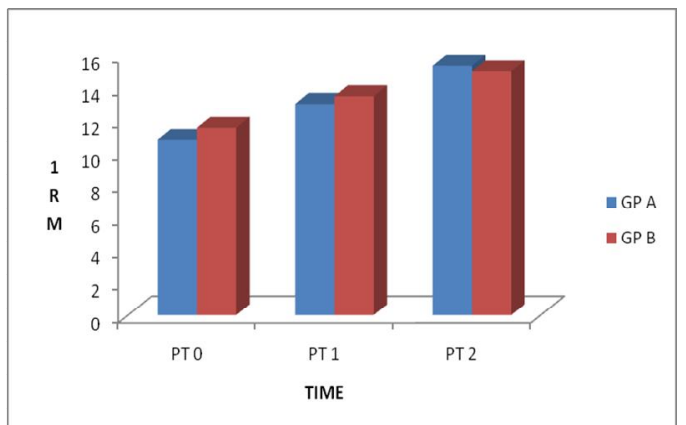

Figure 1. Between Group Analysis of 1 RM 


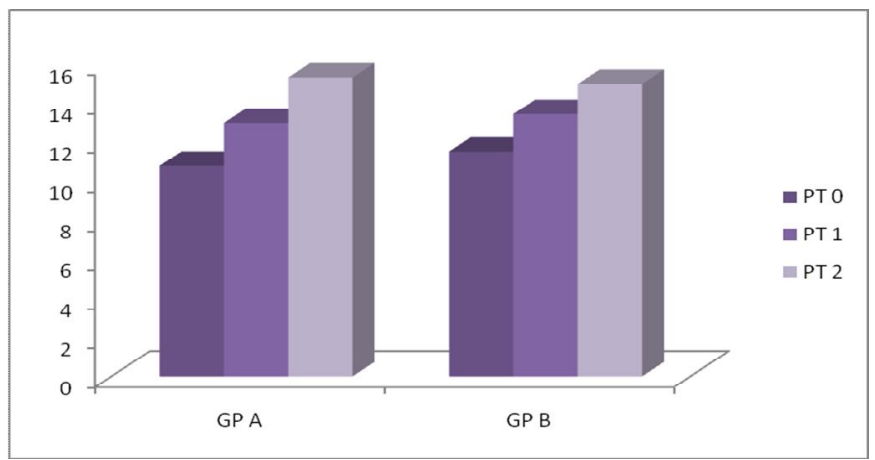

Figure 2.Within Group Analysis of 1 RM

GP A: - Group A ; GP B: - Group B

Comparison of means of pre-test score of $10 \mathrm{RM}$ shows that there is no significant difference between two groups i.e. same at baseline. Comparison of means of $1 \mathrm{RM}$ at 3 weeks and at 6 weeks shows no significant differences. Hence it showed that there is no significant improvement between groups analysis of $10 \mathrm{RM}$. Within group analysis with $10 \mathrm{RM}$ scores shows the significant change in strength when comparison of pre-test to 3 weeks and pre-test to 6 weeks was done. It indicates that the linear periodisation and daily undulating periodisation is effective in improving the strength of quadriceps muscle.

Table 3:- Within and between group comparison for strength $10 \mathrm{RM}$

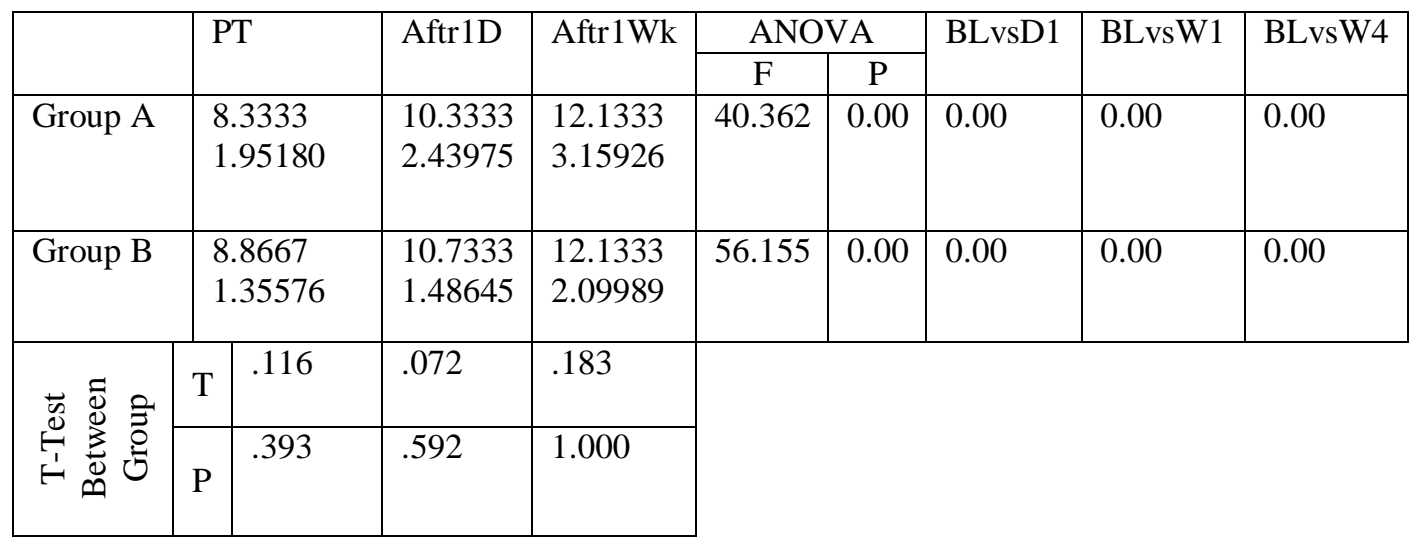

Keys: PT 0: Mean \pm SD of Pre-test scores of $1 \mathrm{RM}$; PT 1: Mean \pm SD of scores after three weeks for 1 RM; PT 2: Mean \pm SD of scores after six weeks for 1RM

Resistance training is becoming an important component of health/fitness programs. The concept of periodization of training has gained greater popularity in the health and fitness arena as it allows for variation in the training stimulus and planned recovery periods to prevent overtraining. Currently, 


\section{Journal of Exercise Science \& Physiotherapy Vol. 15 No. 1 (January to June) 2019 \\ ISSN: 0973-2020 (Print) I2OR Impact Factor =6.502 ISSN: 2454-6089 (Online)}

LP and DUP are two commonly used types of periodization. However, it is unclear which of the two programs elicits greater strength gains. The purpose of this study was to compare linear periodized (LP) and daily undulating (DUP) periodized strength training programs and determine the efficacy of Daily Undulating Periodization over Linear Periodization to improve the strength and endurance of quadriceps in males. This was analyzed by measuring $1 \mathrm{RM}$ and $10 \mathrm{RM}$. To find the effect of LP and DUP to improve the strength and endurance of quadriceps in males of their respective groups, ANOVA was used and to find the efficacy of Daily Undulating Periodization over Linear Periodization over to improve the strength and endurance of quadriceps in normal males, an Independent ' $t$ ' test was used for the study.

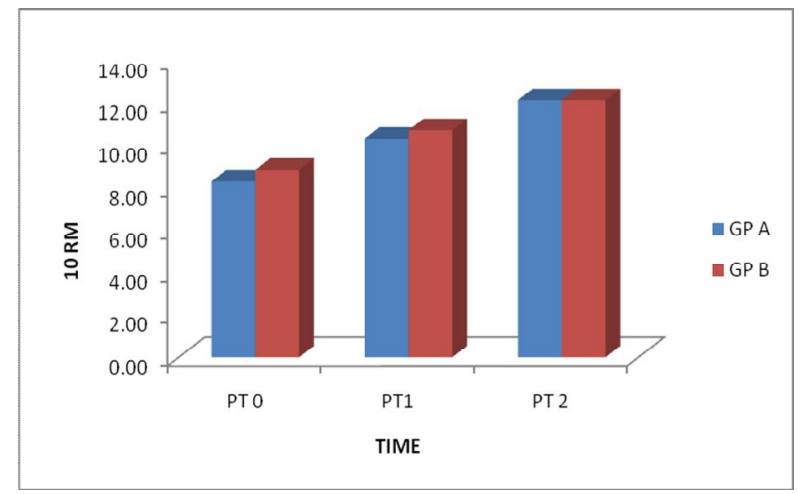

Figure 3. Between Group Analysis of 10 RM

PT 0:- 10 RM score at 0 weeks; PT 1:- 10RM scores at 3 weeks; PT 2:- 10 RM scores at 6 weeks

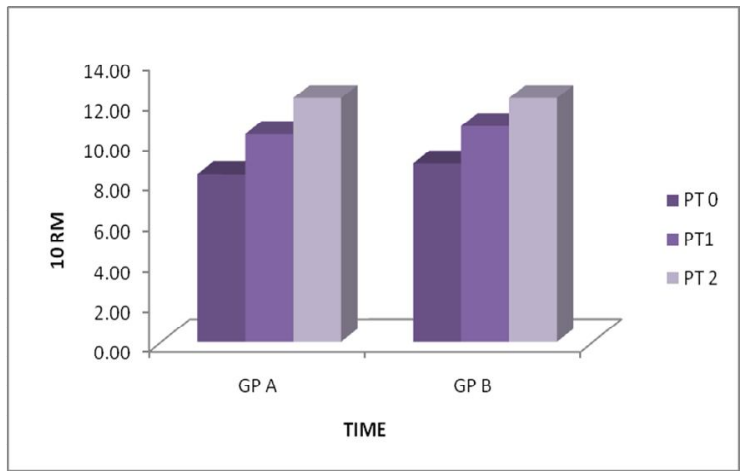

Figure 4. Within Group Analysis of 10 RM

GP A: - Group A ; GP B: - Group B

When the $1 \mathrm{RM}$ and 10RM values to assess the strength and endurance of Quadriceps respectively were analyzed using ANOVA test for significance within the groups, it showed significant improvement individually for both Group-A \& Group-B subjects. When the post-test values of $1 \mathrm{RM}$ and 10RM were subjected to independent ' $\mathrm{t}$ ' test for significance between groups, the calculated value of ' $t$ ' was not significant at $p<0.05$ stating that both the training programs had no 


\section{Journal of Exercise Science \& Physiotherapy Vol. 15 No. 1 (January to June) 2019 ISSN: 0973-2020 (Print) I I OR Impact Factor $=6.502 \quad$ ISSN: 2454-6089 (Online)}

significant variation in the improvement of strength and endurance of quadriceps muscle among the normal males considered for the study, thereby accepting the null hypothesis of the study. The data from the current study suggest DUP and LP are effective methods of eliciting Quadriceps strength and endurance gains for males. However, one method is not superior to the other in eliciting strength and endurance gains during a 6-week training phase. These results support various studies done on athletes and normal untrained individuals in which they reported no significant difference in strength gains between LP and DUP groups when volume and intensity was equated (Pedemonte, J. (1986); Graham, J. (2002); Rhea et al (2002). Research has proven that periodization is an effective method of Resistance training. However, the physiological mechanics behind the effectiveness of DUP and LP and the optimal frequency in which these variables should change is not yet known. The adaptations that occur with the resistance training depend upon how much potential for adaptation exists in the subject, the design of the program followed as well as genetic and psychological factors (Fleck et al (1997). One of the major adaptations that occur with resistance training is the alteration of muscle fiber. Annexure briefs about of the physiological effects or adaptations in the musculoskeletal system that occur with resistance training (Hoffman et al (2003). Neural adaptations that would result are increased recruitment of motor units firing, increased central nervous system activation, lowering of inhibitory reflexes, and inhibition of golgi tendon organs, thus playing an important role in increasing the strength during early training. This often occurs with or without significant changes in muscle size and cross-sectional area. These changes can occur during first 4 weeks of training that has been proved in previous studies in which the strength gain improved to a greater extent during the initial 4 weeks of the training, and it is also evident in the present study. This pattern of strength gain is commonly seen in women as they have less hyper tropic changes due to less testosterone levels. Muscle hypertrophic changes are caused by increased myofibrillar volume that usually occurs during the 4-8 weeks of moderate- high intensity resistance training workouts (Poliquin, C. (1988). There is need of formulating separate program variables for males and females, but to difference in pattern of strength gains for males and females. The results of this study also confirmed age-related reductions in muscle strength and endurance (Brown, A. L. (2001). Similar findings were demonstrated in a study on males (Baker et al (1994). Although if we compare the percentage gain in strength from baseline values, the percent change was greater for the DUP group. These data suggest that DUP provides added variation necessary to elicit maximal strength gains by alternating the volume and intensity of training on a daily basis rather than monthly or weekly (Rhea et al (2002). But the data when subjected to inferential statistics does not reveal any significant differences in the improvement for strength and endurance. Thus, the data from current study states that there is no significant difference in strength and endurance gains in the males trained with LP or DUP protocol. Periodization principles can be used for the normal healthy individuals as well as a part of patient's progression protocol where improvement in physical fitness, strength and endurance are desired.

\section{Conclusion}

The study concludes that both linear periodization and Daily undulating periodization are equally effective means of improving the strength and endurance of Quadriceps in males of age group 1830 yrs.

\section{Acknowledgements}

I am so grateful of the love, support, and encouragement that all of you have provided me along this journey. This project was supported by the Department of Sports Science, Punjabi University Patiala (PB). I would sincerely like to thank all staff members, library staff and my fellow friends for all of their support and encouragement. I would especially like to thank Dr. Anuradha Lehri, Dr. Ashok and Dr. Paramjit for all their input, help, and time. Finally, thank to my entire family member for their continuous support and patience throughout. 


\section{Journal of Exercise Science \& Physiotherapy Vol. 15 No. 1 (January to June) 2019 \\ ISSN: 0973-2020 (Print) I IOR Impact Factor =6.502 ISSN: 2454-6089 (Online)}

\section{References}

Baechle, T. B., \& Earle, R. E. 2000. Essentials of strength training and conditioning. Champaign , IL.

Baker, D., Wilson, G., \& Carlyon, R.1994. Periodization: The effects on strength of manipulating volume and intensity. Journal of Strength and Conditioning Research, 8, 235-242.

Brown, A. L.2001. Nonlinear verses linear periodized models. Journal of Strength and Conditioning Research $23,42-43$

Fleck, S. J., \& Kraemer, W. J. 1997. Designing resistance training programs. Champaign , IL:

Hoffman, J. R., Wendell, M., Cooper, J., \& Kang, J. 2003.Comparison between linear and Non-Linear inseason training programs in freshman football players. Journal of Strength and Conditioning Research, $17,561-565$.

Human Kinetics.Graham, J.2002.Periodization research and example application. National Strength and Conditioning Journal, 24 , 62-70.

Kraemer, W. J. (1997). A Series of Studies- the Physiological Basis for strength training in American Football: Fact over Philosophy. Journal of Strength and Conditioning Research, 11, 131-142.

Pedemonte, J. (1986). Foundations of training periodization part I: Historical outline. Strength and Conditioning Journal, 8 , 62-65.

Poliquin, C. (1988). Five steps to increasing the effectiveness of your strength training program. Journal of Strength and Conditioning Research, 10, 34-39.

Rhea, M. R., Ball, S. D., Phillips, W. T., \& Burkett, L. N. (2002). A comparison of linear and daily undulating periodized programs with equated volume and intensity for strength. Journal of Strength and Conditioning Research, 16 , 250-255.

Stone, M. H., O'Bryant, H., \&Garhammer, J. (1981). A hypothetical model for strength training. Journal of Sports Medicine Physical Fitness, 21 , 342-351.

Conflict of Interest: None declared 